\title{
Scintillation Loss in Optical Low Earth Orbit Data Downlinks with Avalanche Photodiode Receivers
}

\author{
Dirk Giggenbach \\ Florian Moll
}

\author{
Institute of Communications and Navigation \\ German Aerospace Center \\ 82234 Wessling, Germany
}

\begin{abstract}
Future Earth Observation satellite data downlinks via directed laser beams are a promising technique to overcome the data repatriation bottleneck in high resolution sensor missions. A challenge for this technology are received power scintillations induced by atmospheric index-of-refraction turbulence (IRT). The strength of these signal variations depends on the IRT height profile, receiver aperture size, wavelength, and link elevation. We analyze measured optical satellite downlink power vectors to verify a model for the dynamic power scintillation loss. Due to their increased sensitivity compared with PIN-receivers, Avalanche Photo Diodes (APD) are usually used within data receivers on ground. This allows bulk receivers to avoid the demanding coupling onto single-mode detectors. As their sensitivity run is defined by the influence of thermal-, and signal-dependent shot-noise, a new formulation for this dependency is evaluated, and the according modified scintillation loss is quantified. This allows appropriate dimensioning of link budgets and the necessary error control mechanisms to enable reliable high-speed data transfer from low earth orbit satellites to the ground.
\end{abstract}

Keywords - FSO, optical LEO downlinks, scintillation loss, shot-noise limited receiver, Avalanche Photo Diode

\section{INTRODUCTION}

Optical telemetry downlinks from low Earth orbit (LEO) observation satellites can enable the required growth in remote sensor data throughput. According technology has been demonstrated in experiments [1][2][3] and is currently being developed and standardized, based on direct detection optoelectrical conversion in the optical ground station (OGS) [4][5]. The Earth's atmosphere distorts the signal wave-front dynamically by index-of-refraction turbulence (IRT), leading to amplitude variations (intensity speckles) and phase front distortions, as well known from according theory and measurements [6][7][8][9]. When employing direct detection reception with multi-mode photo receivers, no correction of the phase aberrations is necessary in a first approach. Then moderately sized ground telescope apertures can be employed, integrating over the intensity speckle structure size. With this so-called aperture averaging effect, the strength of received power fades as well as their duration is reduced from the stronger variations in intensity, adding to the beneficial inherent increase in average receive power with aperture area. This reduces the urge for alternative fading-compensation techniques, namely long data interleavers together with strong forward error correction coding. On the other side, keeping the aperture as small as required - governed basically by the static link budget - will reduce costs and other SWaP constraints (Size, Weight and Power) for the OGS. Thus the correct estimation of the aperture averaging effect at all link elevations of interest and for a given OGS aperture diameter is of paramount importance to ensure the required data reception quality in optical LEO direct-to-Earth (DTE) data downlinks.

Link duration, range, and angular slew rate for optical LEO-DTE links are as known from conventional EO-satellite downlinks, where we assume a minimum elevation of $5^{\circ}$ for the initial contact between satellite and OGS, and $10^{\circ}$ for stable communications.

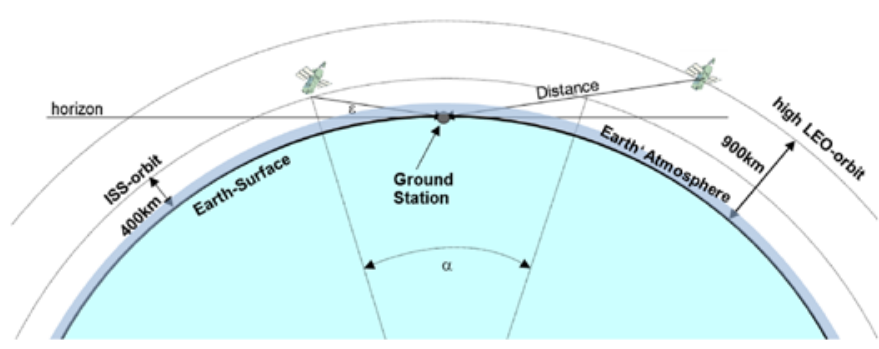

Fig. 1. Geometrical constraints in Direct-to-Earth links for a very low orbit (taking as example the ISS) and a high LEO.

However the PAA (point-ahead angle) is an additional parameter, indicating the angle between incoming downlink and upwards uplink beam caused by the satellite's movement during the signal round trip time. This PAA can become significantly larger than diffraction limited beam diameters and also varies during the link, see TABLE I. Therefore, downwards and upwards beam pointing direction must take the PAA into account. 
TABLE I. GEOMETRICAL PARAMETERS FOR LEO-DTE

\begin{tabular}{|c|c|c|c|c|}
\hline \multirow[b]{2}{*}{ Orbit } & \multicolumn{4}{|c|}{ Parameters } \\
\hline & $\begin{array}{c}\text { Distance } \\
\text { at } 5^{\circ}\end{array}$ & $\begin{array}{c}\text { Max. link } \\
\text { duration } 5^{\circ}-5^{\circ}\end{array}$ & $\begin{array}{c}\text { Slew-rate at } \\
\text { zenith }\end{array}$ & $\begin{array}{l}\text { Point-ahead at } \\
\text { zenith (polar } \\
\text { orbit) }\end{array}$ \\
\hline $400 \mathrm{~km}$ & $1804 \mathrm{~km}$ & $475 \mathrm{~s}$ & $1.1 \% \mathrm{~s}$ & $51 \mu \mathrm{rad}$ \\
\hline $900 \mathrm{~km}$ & $2992 \mathrm{~km}$ & $831 \mathrm{~s}$ & $0.48 \% \mathrm{~s}$ & $49 \mu \mathrm{rad}$ \\
\hline
\end{tabular}

The importance of low elevations is indicated by their total visibility time fraction: When we e.g. assume stable data reception from $10^{\circ}$ elevation upwards, the fraction between $10^{\circ}$ and $20^{\circ}$ accounts for half of the total visibility above $10^{\circ}$, see Fig. 2. It is therefore of major importance that the downlink techniques can cope with the strong IRT and other losses at low elevations.

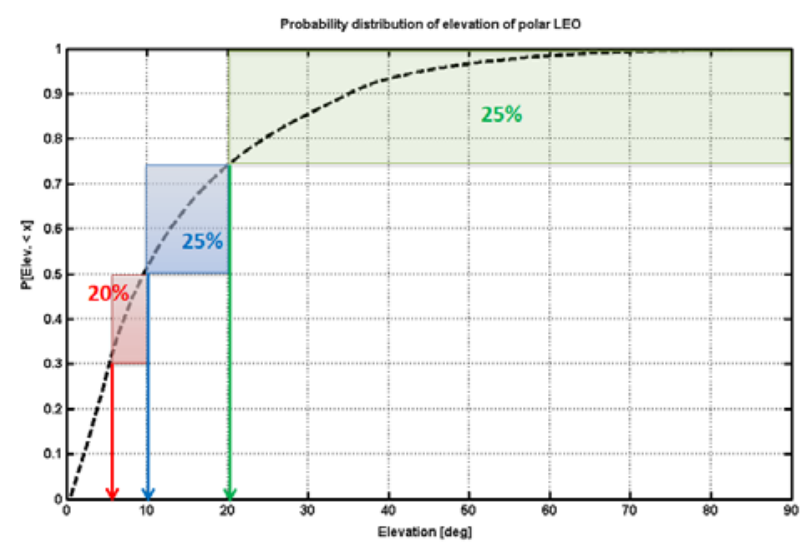

Fig. 2. Fraction of total satellite visibility above horizon versus elevation, for a typical earth observation satellite (polar orbit at $600 \mathrm{~km}$ height), the relative curve-shape is similar to any OGS-location on earth.

The received power at the OGS experiences fades and surges with typical durations in the millisecond scale. Figure 3 shows an exemplary run of received power over elevation at nighttime (intermittent outages are caused by tracking failures). The approx. constant absolute min-max span over elevations (black dots) - while the mean received power is increasing with decreasing range - is typically observed in the OLEO-DTE scenario.

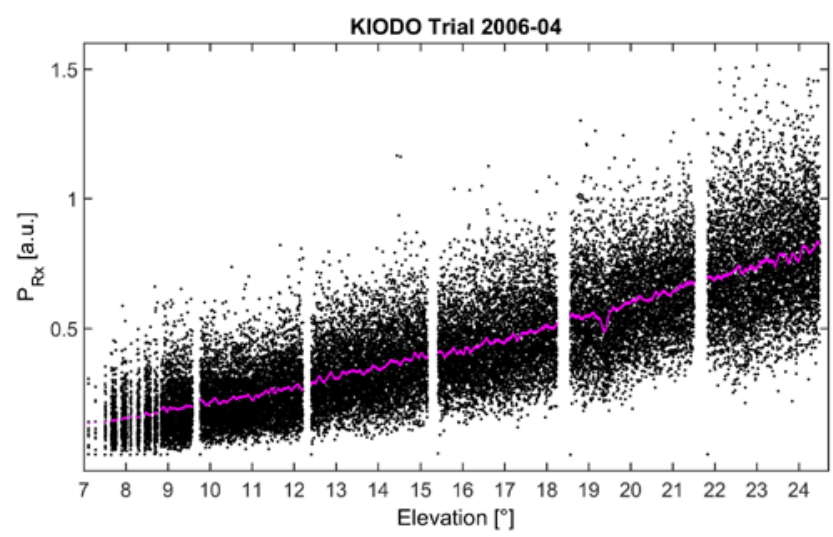

Fig. 3. Received power as seen by a $40 \mathrm{~cm}$ telescope. Signal wavelength is $847 \mathrm{~nm}[16]$

\section{POWER SCINTILLATION MODELling}

A mathematical description of an optical wireless transmission system with direct detection receiver and output $Y$ through an atmospherically distorted fading channel is

$$
Y=A \cdot X+N(A)+N_{c},
$$

where the vector $X$ represents the transmitted data symbols, $N_{c}$ the signal-independent and $N(A)$ the signal-dependent noise. $A$ is the received signal amplitude given through transmit power, divergence, attenuation and distance, $A$ is changing over elevation and varies due to fast shifts in the turbulent index-ofrefraction. $Y$ represents the received signal deteriorated by noise and fading. In a direct-detection receiver with the electrical output amplitude linear to optical input power, the signal-dependent noise component $N(A)$ leads to a non-linear relation of the receiver-SNR versus $A$ [26].

The variability of the optical intensity when an optical beam propagates through perturbing atmospheric turbulence is expressed by the intensity scintillation index ISI (normalized variance of the optical intensity at time $t$ and location $p$ )

$$
\sigma_{I}^{2}=\frac{\operatorname{VAR}[I(t, p)]}{E[I(t, p)]^{2}}
$$

In weak-turbulence, $\sigma_{I}^{2}$ can be approximated by the RytovIndex (RI) $\sigma_{R}{ }^{2}$ which can be derived from the turbulence strength $C_{n}^{2}$ along the propagation path by (3), where the slant link path above a spherical earth surface must be taken into account to calculate $h(z)$ and the maximum distance $Z$ at maximum height $H$

$$
\sigma_{R}^{2}=\frac{19.2}{\lambda^{7 / 6}} \cdot \int_{z=0}^{Z(H)} C_{n}^{2}(h(z)) \cdot z^{5 / 6} d z
$$

A realistic height profile of the index of refraction structure constant $C_{n}^{2}(h)$ is required to model the IRT along the link. While the classical Hufnagel-Valley (HV) model cannot account for the ground turbulence effect above an OGS which is at an altitude higher than sea-level, the HV-Modified (HVM) [17] regards this situation by increasing the profile towards the ground station altitude $H_{O G S}$, while it maintains the HV-profile at high altitudes above the OGS as referred to sea-level. Sea-level referenced $A_{0}$ at daytime is typically estimated as $3 \mathrm{E}-13 \mathrm{~m}^{-2 / 3}$ and as $1.7 \mathrm{E}-14 \mathrm{~m}^{-2 / 3}$ at nighttime, while as wind speed at the tropopause $v$ we assume $10 \mathrm{~m} / \mathrm{s}$. 


$$
\begin{aligned}
& C_{n}^{2}(h)=5.94 \cdot 10^{-3} \cdot\left(\frac{v}{21 \frac{m}{s}}\right)^{2} \cdot\left(10^{-5} \cdot h / m\right)^{10} \cdot e^{-h / 1000}+ \\
& +2.7 E-16 \cdot e^{-h / 1500 m}+A_{0} \cdot e^{-H_{O C S} / 700 m} \cdot e^{-h-H_{O C S} / 100 m}
\end{aligned}
$$

Some examples of this profile are depicted in Fig. 4. The spherical Earth model is taken into account in all further evaluation to derive $h(z)$.

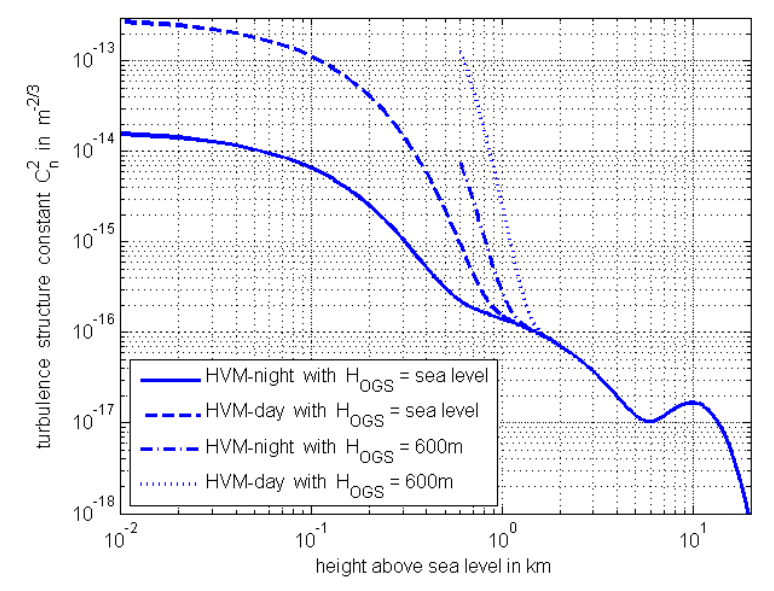

Fig. 4. Hufnagel-Valley-Modified (HVM) used here to approximate $C_{n}^{2}(h)$, for OGS located at sea level and at $H_{O G S}=600 \mathrm{~m}$ and as $A_{0}=1.7 \mathrm{E}-14 \mathrm{~m}^{-2 / 3}$ (night) and $A_{0}=3 \mathrm{E}-13 \mathrm{~m}^{-2 / 3}$ (day). For $H_{O G S}=$ sea level the HVM coincides with the classical HV.

In optical DTE communications, finite-sized ground receiver apertures must be employed. Then their aperture averaging effect relates the intensity scintillation index to the received power scintillation index (PSI) with aperture diameter $D$ by

$$
A_{f}(D)=\frac{\sigma_{P}^{2}(D)}{\sigma_{I}^{2}}
$$

Estimation of $A_{f}$ is done by according methodologies based on the spatial intensity distribution [18][19][20], where an approximation for plane wave and weak turbulence is based on the intensity structure size parameter $\rho_{I}$ :

$$
A_{f}=\left[1+1.062\left(\frac{D}{2 \rho_{I}}\right)^{2}\right]^{-7 / 6}
$$

This $\rho_{I}$ is derived in a rough approximation through the Fresnel-size given by the distance $L$ from a dominant turbulent layer (or with more effort and uncertainty by estimating the intensity covariance structure function from the $C_{n}^{2}$-profile). This layer is assumed as the tropopause at $H_{d}=12 \mathrm{~km}$ analog to [21]. We here extend the modelling to the peaking and reduction of the structure size below a maximum-size elevation $\varepsilon_{\max } \sim 10^{\circ}$

$$
\rho_{I} \approx 1.5 \cdot \sqrt{\frac{L \cdot \lambda}{2 \pi}} \quad ; \quad L \approx H_{d} \frac{\varepsilon / 90^{\circ}}{\left(\varepsilon / 90^{\circ}\right)^{2}+\left(\varepsilon_{\max } / 90^{\circ}\right)^{2}}
$$

Albeit (6) and (7) are rough approximations valid only in the weak turbulence regime (neglecting multiple scattering and scintillation saturation in long atmospheric paths as found at very low elevations), the resulting modelling of PSI shows good accordance with measured values as can be seen in Fig. 5 and Fig. 6. It even performs better than employing allregime approximation functions which are but limited to horizontal paths and zero inner scale. An experimental investigation of $\rho_{I}$ through analysis of the intensity covariancefunction, together with extended Rytov theory, can be found in [8]. Since here we limit minimum elevations to $10^{\circ}$, the described modelling proofs sufficiently accurate.

\section{PSI MOdELl COMPARED to MEASUREMENTS}

Here the consistency of measured PSI data with values generated by (3) through (7) is shown. Measurements were carried out with power detectors in the $40 \mathrm{~cm}$ telescope of Optical Ground Station Oberpfaffenhofen (OGSOP), and into a $5 \mathrm{~cm}$ aperture parallel to the main telescope. Additionally, intensity statistics were derived from the single pixel values of a pupil camera behind the main telescope. A description of the telescope and the measurement system can be found in [10],[11]. The downlink trials employed the collimated beam from OICETS at $847 \mathrm{~nm}$ signal wavelength, performed in 2006 [16] and 2009. For $1550 \mathrm{~nm}$ more divergent beams from the OPALS terminal on the ISS and SOTA onboard SOCRATES [12],[13],[14],[15] were used.

Fig. 5 compares theoretical RI (weak turbulence approximation) and PSI according to the described model with measured ISI and PSI samples, into $40 \mathrm{~cm}$ for $847 \mathrm{~nm}$ and OGS at $600 \mathrm{~m}$ above sea level. Fig. 6 does the same with downlinks at $1550 \mathrm{~nm}$ wavelength. The HVM $C_{n}{ }^{2}$-profile fits well to the measured parameters with $A_{0}=1.7 \mathrm{E}-14 \mathrm{~m}^{-2 / 3}$ and $\mathrm{v}=10 \mathrm{~m} / \mathrm{s}$ for the $847 \mathrm{~nm}$ downlinks (which all took place during the middle of the night) and with $A_{0}=1 \mathrm{E}-13 \mathrm{~m}^{-2 / 3}$ and $\mathrm{v}=10 \mathrm{~m} / \mathrm{s}$ for the $1550 \mathrm{~nm}$ experiments. 


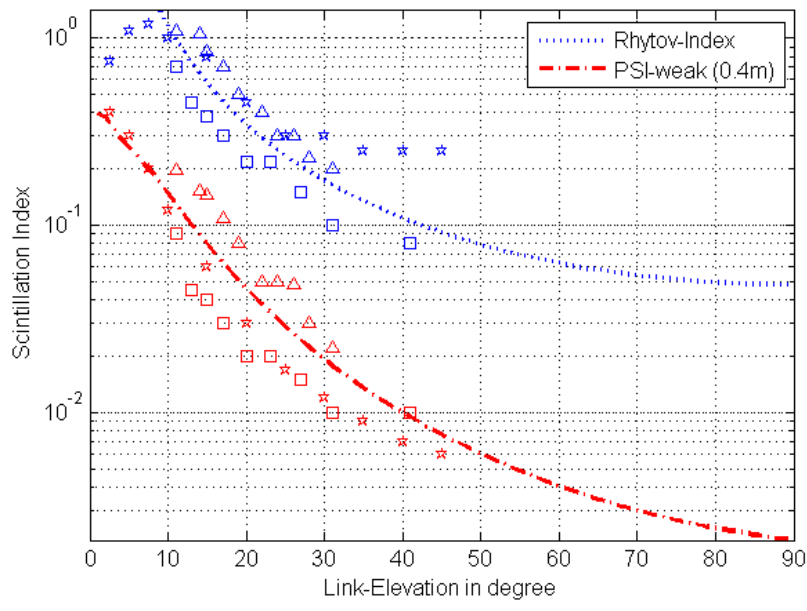

Fig. 5. Analytical and measured ISI (point receiver) and PSI for $40 \mathrm{~cm}$ aperture size at $847 \mathrm{~nm}$.

Modell and measurements coincide down to $10^{\circ}$ elevation, below the scintillation saturation leads to discrepancies with intensity scintillation, but PSI modelling still fits well.

Scintillation data for $1550 \mathrm{~nm}$ wavelength with SOTA and OPALS taken at OGS-OP in 2015 and 2016 is compared with the calculated curves in Fig. 6.

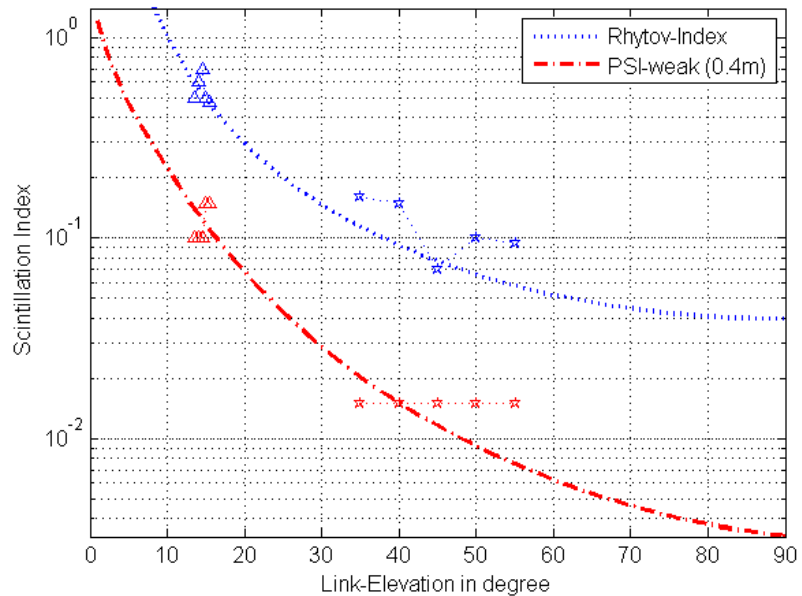

Fig. 6. Same as Fig. 5 but for 1550 nm, representing ISI and PSI measurements from OPALS and SOTA.

\section{Scintillation LOSS FOR AN Optical POWER Detector}

Under a certain amount of aperture averaging the $\mathrm{Rx}$ power probability density function (PDF) is the sum of several random intensity values impinging on the aperture in parallel, which for increasing apertures diameters evolves via a lognormal towards a normal distribution.

The lognormality of the distribution is in agreement with the findings in [22] where aperture averaging was analyzed with wave optics simulations. It was found that lognormality can be seen for the case of aperture sizes larger than the Fresnel zone in weak scintillation conditions (here defined as $\sigma_{R}{ }^{2}<0.55$ ) and in strong scintillation conditions when the aperture is larger than the coherence width. In moderate scintillation strength $\left(0.55<\sigma_{R}{ }^{2}<5.45\right)$, the PDF rather resembles a gamma-gamma shape. Vetelino et al. report in [23] that in the moderate-to-strong fluctuation regime the PDF of apertures larger or equal to the coherence radius is lognormal. These finding are supported by analysis of fade statistics by simulation and analytical investigation in [24]. Experimental investigation of aperture averaging in the strong fluctuation regime is described in [25] and fits to the observation that aperture sizes larger than the coherence radius produce a lognormal shape.

Thus, lognormality of the statistics can be assumed and the PDF is expressed with the power scintillation index $\sigma_{P}{ }^{2}$ and the mean power $P_{0}$ as:

$$
p\left(P, \sigma_{P}^{2}\right)==\frac{1}{P \cdot \sqrt{2 \pi \cdot \ln \left(\sigma_{P}^{2}+1\right)}} \cdot e^{-\frac{\left(\ln \frac{P}{P_{0}}+\frac{1}{2} \ln \left(\sigma_{P}^{2}+1\right)\right)^{2}}{2 \cdot \ln \left(\sigma_{P}^{2}+1\right)}}
$$

Fig. 7 shows measured received power PDFs under various elevation angles from KIODO downlink vectors and illustrates their affinity to lognormal distributions.

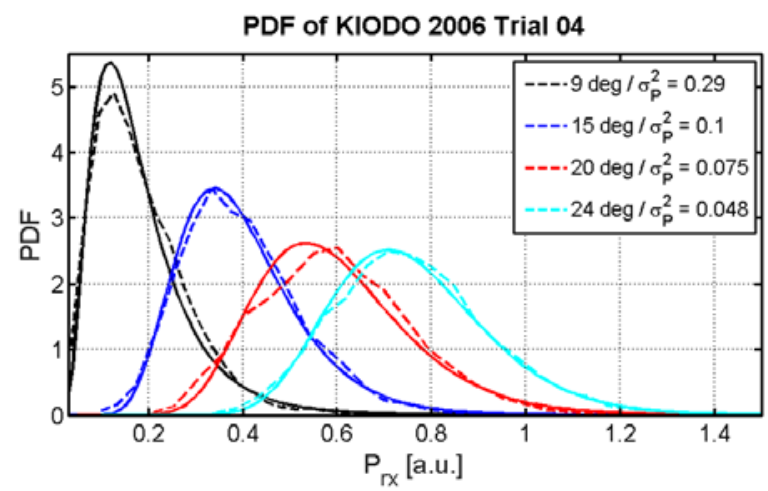

Fig. 7. Probability density measurements of received power at various elevations for a $40 \mathrm{~cm}$ receiver aperture are confirmed as lognormally distributed by their analytical fits. Measured during KIODO at $847 \mathrm{~nm}$ wavelength.

The loss-fraction $p_{t h r}$ is the share where the received power falls below a given threshold from mean power. It is assumed that data cannot be detected during these fades - the channel is thus modelled by on/off states. The data segments lost during these fading states have to be recovered by according means like channel coding together with interleaving, as it is standard practice in free-space and wired data communications today.

The according derivation for the loss $a_{s c i}$ in $\mathrm{dB}$ with a lognormal power PDF according to [27] is repeated here: 


$$
a_{s c i}=-4.343\left[\begin{array}{l}
\operatorname{erfinv}\left(2 p_{t h r}-1\right) \sqrt{2 \ln \left(\sigma_{P}{ }^{2}+1\right)}- \\
-\frac{1}{2} \ln \left({\sigma_{P}}^{2}+1\right)
\end{array}\right]
$$

The principle is illustrated in Fig. 8 with mean power, the loss fraction $p_{t h r}$ as integral of the PDF-tail, and the according loss.

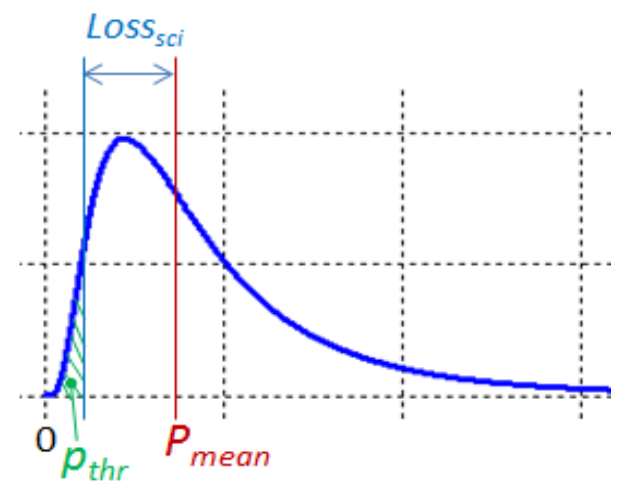

Fig. 8. Deriving scintillation loss in a lognormal fading channel, relative to mean power $P_{\text {mean }}$ and with loss-fraction $p_{t h r}$., illustrated with the power vector's probability distribution (blue line).

When assuming a receiver with signal-independent noise $N_{C}$ only (i.e. $N(A)=0$ ), with (9) here we calculate the loss for $10 \mathrm{~cm}$ and $40 \mathrm{~cm}$ aperture and for loss fractions of $10^{-6}$ and $1 \%$ (as can be recovered by state-of-the-art forward error correction coding with interleaving), as shown in Fig. 9 and Fig. 10. This time we set the same turbulence profile for both wavelengths for better comparison, with $A_{0}=1.7 \mathrm{E}-14 \mathrm{~m}^{-2 / 3}$ and $v=10 \mathrm{~m} / \mathrm{s}$. We limit the display to a minimum elevation of $10^{\circ}$ since our model is not verified below.

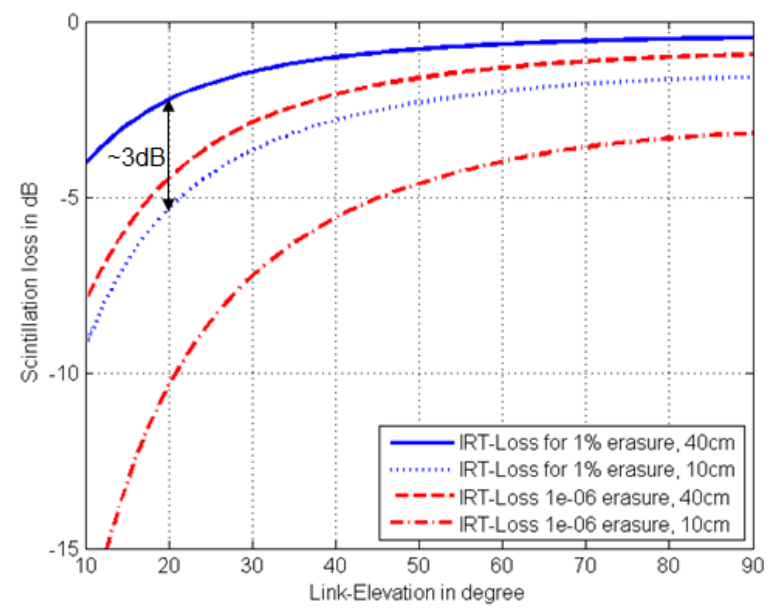

Fig. 9. Scintillation loss in OLEODL for thermal-limited power detectors at $847 \mathrm{~nm}$ for $10 \mathrm{~cm}$ and $40 \mathrm{~cm}$ receiver telescope and loss fractions of $1 \%$ and $10^{-6}$. The relative advantage by aperture averaging of the $40 \mathrm{~cm}$ versus a $10 \mathrm{~cm}$ aperture is approx. $3 \mathrm{~dB}$ for $1 \%$ loss fraction, and more for the smaller loss fraction $10^{-6}$.

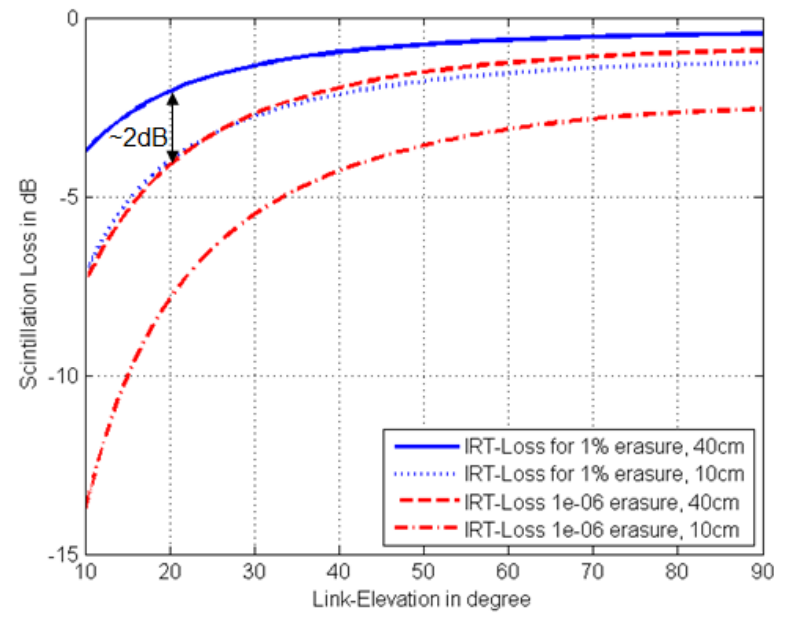

Fig. 10. Same as Fig. 9 but for $1550 \mathrm{~nm}$.

The gain by aperture averaging of $40 \mathrm{~cm}$ versus $10 \mathrm{~cm}$ receiver diameter is indicated by arrows for an elevation of $20^{\circ}$. This gain reduces with longer wavelengths, i.e. shorter wavelengths do benefit more from the aperture averaging effect. Obviously, atmospheric scintillation can lead to strong losses with small apertures and under low elevations, while with fairly large telescope apertures (approx. more than $1 \mathrm{~m}$ ) they tend to become negligible - assuming that no pointingjitter is adding to downlink fading.

\section{LOSS WITH GENERALIZED RECEIVER-MODEL}

The influence of optical power onto the received electrical signal quality at the output of a receiver frontend (RFE) is generally given by the characteristics of the opto-electric detector. The latter is defined by its $Q$-factor (quality-factor) which is related to other practical IM/DD RFE-parameters as

$$
B E R=\frac{1}{2} \cdot \operatorname{erfc}\left(\frac{Q}{\sqrt{2}}\right) ; \quad Q=\sqrt{S N R_{e l}}=f\left(\bar{P}_{R x}\right)
$$

where Bit Error Ratio (BER) is generally determining transmission quality, effectively equivalent to $Q$. For a good APD-receiver $Q$ should be dominated by signal-dependent shot-noise during reception of a binary " 1 " $\left(\sigma_{s, 1}\right)$, and not by the constant thermal noise $\left(\sigma_{t}\right)$ as in PINs. The approximate modelling of different types of optical receivers can be done with a simple exponential function:

$$
Q\left(\bar{P}_{R x}\right)=\left(\frac{\bar{P}_{R x}}{\bar{P}_{Q=2}}\right)^{n}
$$

With exponent $n$ defining receiver sensitivity slope and with absolute sensitivity $P_{Q=2}$. 
High quality InGaAs APD-receiver implementations show a normalized sensitivity for $Q=2$ of around 20 aJ per bit and exhibit a sensitivity-run close to the square-root of received power. PIN receivers instead show a linear dependency. For further details on the concept see [26].

Values of $n$ for thermal-limited and coherent receivers are typically close to their theoretical values 1 and 0.5 respectively, while for APD-RFEs the exponent can vary strongly, dependent on specific semiconductor and electronic circuit implementation. Typical values are shown in Fig. 11 and TABLE II. Their run normalized to $P_{Q=2}$ is shown in Fig. 12.

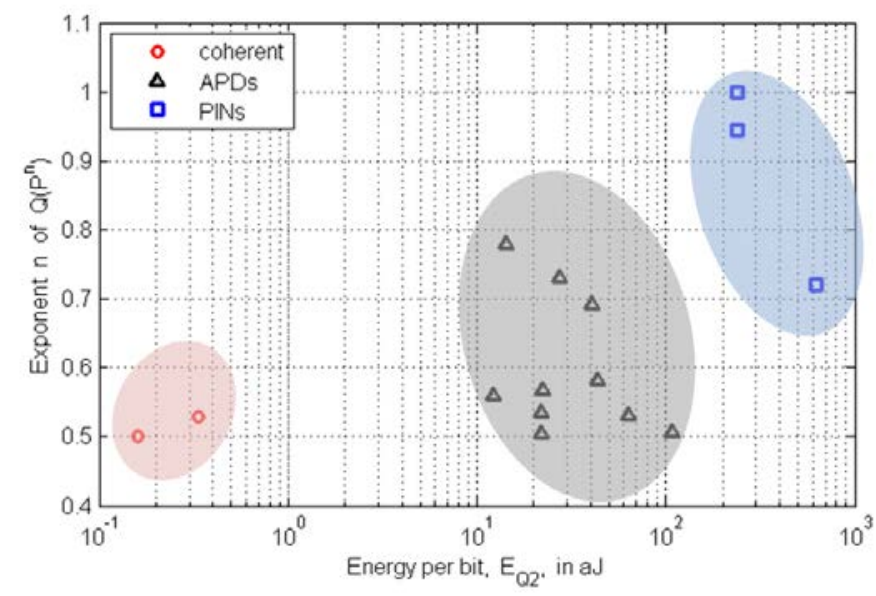

Fig. 11. Typicall RFE sensitivity parameter ranges with rate-normalized reciver sensitivity in energy/bit, showing required bit-energy for $Q=2$ (i.e. $\mathrm{BER}=0.023$ ) and slope-exponent $n$. Marked in gray is the value range for APD-receivers.

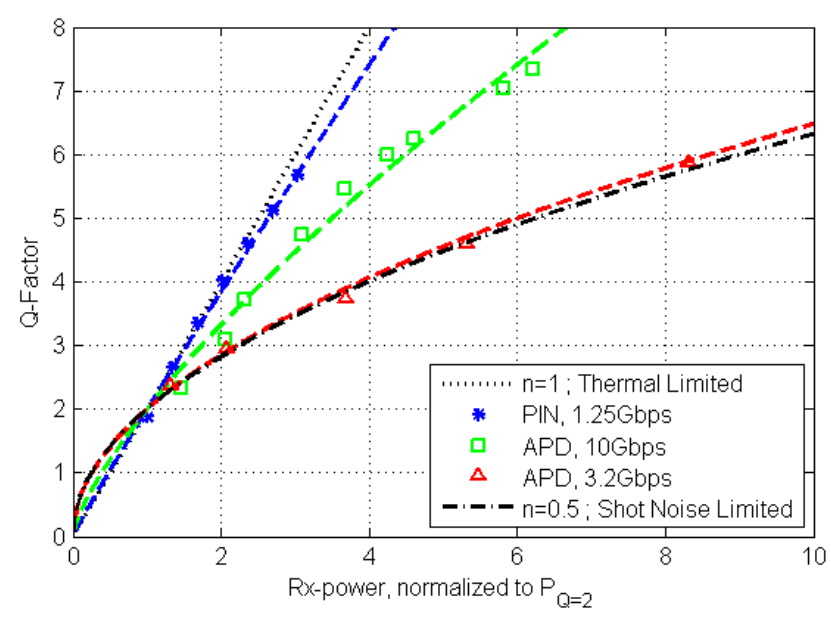

Fig. 12. Exemplary runs of $Q\left(\bar{P}_{R x}\right)$ normalized to $\bar{P}_{Q=2}$.
TABLE II. TYPICAL MEASURED VALUES OF RFE-SENSITIVITIES AS CAN BE USED TO MODEL PRACTICAL RFE BEHAVIOUR

\begin{tabular}{|c|l|c|c|}
\hline \multirow{2}{*}{ Modulation } & \multicolumn{3}{|c|}{ Parameters } \\
\cline { 2 - 4 } & \multicolumn{1}{|c|}{ RFE-Specification } & $\boldsymbol{P}_{\mathbf{Q}=2}$ & $\boldsymbol{n}$ \\
\hline IM/DD & $\begin{array}{l}\text { 1.25Gbps InGaAs-PIN (thermal } \\
\text { lim.) at 1550nm }\end{array}$ & $296 \mathrm{nW}$ & 0.95 \\
\hline IM/DD & $\begin{array}{l}\text { 10Gbps InGaAs-APD with } \\
\text { medium } n \text { at 1550nm }\end{array}$ & $273 \mathrm{nW}$ & 0.73 \\
\hline IM/DD & $\begin{array}{l}\text { 3.2Gbps InGaAs-APD with } \\
\text { low } n \text { at 1550nm }\end{array}$ & $71.2 \mathrm{nW}$ & 0.51 \\
\hline BPSK & $\begin{array}{l}\text { 2Mbps Balanced coherent } \\
\text { homodyne Rx at 1064nm }\end{array}$ & $672 \mathrm{pW}$ & 0.53 \\
\hline
\end{tabular}

Taking into account the preceding description, we must modify the derivation of scintillation loss from the simple linear-to-power model as in section IV to a generalized loss:

The exponent $n$ models the dependency of $Q$ versus the received optical power $P$, and we derive the modified probability density function of $Q$ as a function of the normaldistributed lognorm-amplitude $\chi$ :

$$
f_{p}\left(Q, n, \sigma_{\chi}^{2}\right)=\frac{1}{2 n Q \sqrt{2 \pi \cdot \sigma_{\chi}^{2}}} \cdot \exp \left[-\frac{\left[\ln \frac{Q}{\langle Q\rangle}+2 n \cdot \sigma_{\chi}^{2}\right.}{8 n^{2} \cdot \sigma_{\chi}^{2}}\right]
$$

with $\langle\chi\rangle=-\sigma_{\chi}{ }^{2}$ as applicable for lossless lognormal scintillation, where $\chi$ relates to the PSI of received power $\sigma_{P}{ }^{2}$ as $\sigma_{P}^{2}=e^{4 \sigma_{\chi}^{2}}-1$ [6].

The QSI (signal-Quality Scintillation Index) $\sigma_{Q}{ }^{2}$ (derived analog to ${\sigma_{P}}^{2}$ as the normalized variance of $Q$ ) then takes a rather simple general term depending on ${\sigma_{P}}^{2}$ :

$$
\sigma_{Q}^{2}\left(\sigma_{P}^{2}, n\right)=\left(\sigma_{P}^{2}+1\right)^{n^{2}}-1
$$

Thus $\sigma_{Q}{ }^{2}$ will practically have a value between $\sigma_{P}{ }^{2}$ (for $\mathrm{n}=1$ ) and smaller than $1 / 4 \sigma_{P}^{2}$ (for $n=0.5$ ). Since the distribution function will again follow a lognormal behavior, the general scintillation loss $a_{\text {sci-Q }}$ in $\mathrm{dB}$ can be deduced as simply the power scintillation loss from (9) with the additional factor $n$ :

$$
a_{s c i-Q}\left(\sigma_{P}^{2}, n, p_{t h r}\right)=n \cdot a_{s c i}\left(\sigma_{P}^{2}, p_{t h r}\right)
$$

Fig. 13 compares this general loss in $Q$ for the practical 10Gbps APD-RFE ( $n=0.73$ ) with the two theoretical RFEs (thermal- and shot-noise limited), at daytime and $H_{O G S}=600 \mathrm{~m}$. 


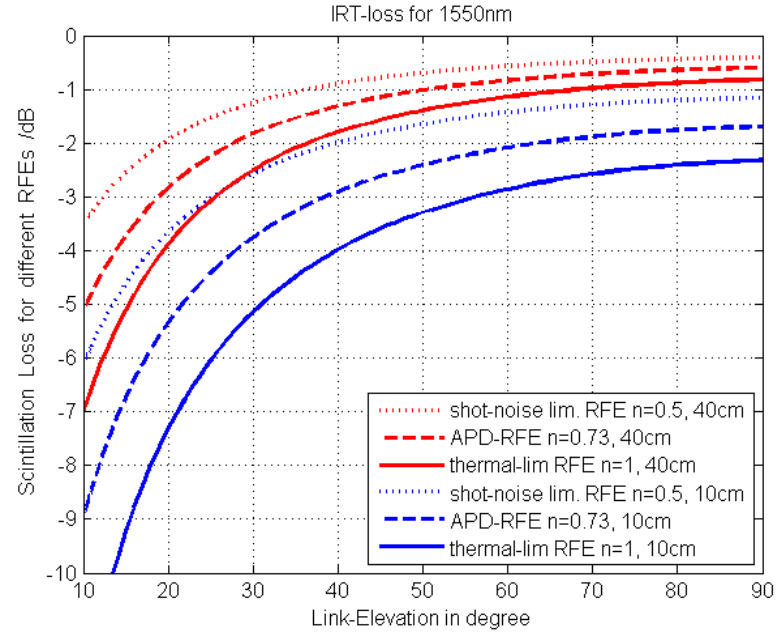

Fig. 13. Relative Scintillation-Loss in $Q$ at $1550 \mathrm{~nm}$ with loss-fraction $1 \%$, for three different receiver types and for $40 \mathrm{~cm}$ and $10 \mathrm{~cm}$ telescope diameter.

The advantage of APDs versus thermal-limited power detectors becomes obvious: The scintillation loss can be reduced to below $3.3 \mathrm{~dB}$ with a shot-noise limited receiver at $10^{\circ}$ elevation and with $40 \mathrm{~cm}$ aperture, and to below $5 \mathrm{~dB}$ with our exemplary 10 Gbps APD-RFE, while the thermal-limited receiver shows $7 \mathrm{~dB}$ loss. The achievable gain of an RFE with signal-dependent noise is higher for the smaller aperture, e.g. a $10 \mathrm{~cm}$ aperture has roughly twice the scintillation loss of a $40 \mathrm{~cm}$ telescope in $\mathrm{dB}$.

Note that the absolute receiver sensitivity (e.g. $\bar{P}_{Q=2}$ ) is not relevant for the relative scintillation loss in (14), however must be taken into account for an absolute link budget estimation.

\section{SUMMARY}

We derive a model for the power scintillation-index and loss in optical LEO satellite downlinks, over elevation and for arbitrary wavelengths and telescope diameters. Introducing according day- or night-time $C_{n}^{2}$-profiles and choosing the receiver frontend sensitivity characteristics, one derives the scintillation loss for a given loss-fraction. The scintillation model is confirmed with various measurements at $847 \mathrm{~nm}$ and $1550 \mathrm{~nm}$ signal wavelength, down to $10^{\circ}$ link elevation. This allows the appropriate design of error-correction techniques to protect the data transfer against fading outages.

As one result we find that the scintillation loss for $1550 \mathrm{~nm}$ does not differ significantly from $847 \mathrm{~nm}$, mainly since the lower scintillation strength with longer wavelength is partly counteracted by the lower aperture averaging effect as caused by the larger intensity structure sizes. Only for very small apertures (smaller than intensity structure $\rho_{I}$ ) the advantage of longer wavelengths would become significant. However, we assume Rx-apertures smaller than the intensity speckle structures are not applicable in OLEO-DTE due to link budget constraints
A new model for assessment of scintillation loss taking into account different receiver types is developed. We verify the advantage of practical APD-receivers compared to thermal noise limited power detectors, which for $40 \mathrm{~cm}$ aperture reduces the scintillation loss by several $\mathrm{dB}$, depending on receiver sensitivity run, aperture size, wavelength, and IRTprofile. This beneficial behavior of APDs adds significantly to their anyway increased absolute sensitivity over pure PIN power detectors.

\section{ACKNOWLEDGMENT}

We gratefully acknowledge JAXA, NICT, and NASA-JPL for enabling the optical LEO downlinks from OICETS, SOCRATES/SOTA, and OPALS, to OGS-OP. We thank our colleague Gianluigi Liva for his review of the concept presented in this paper.

\section{REFERENCES}

[1] N. Perlot, M. Knapek, D. Giggenbach, J. Horwath, M. Brechtelsbauer, Y. Takayama, T. Jono, "Results of the Optical Downlink Experiment KIODO from OICETS Satellite to Optical Ground Station Oberpfaffenhofen (OGS-OP)”, Proc. of the SPIE -- Volume 6457, 2007

[2] H. Takenaka, Y. Koyama, D. Kolev, et al., "In-orbit verification of small optical transponder (SOTA) -Evaluation of satellite-to-ground laser communication links”, Proc. of SPIE 9739, 2016

[3] M.J. Abrahamson, B.V. Oaida, O. Sindiy, A Biswas, "Achieving operational two-way laser acquisition for OPALS payload on the International Space Station ", Proc. SPIE 9354, 2015

[4] Giggenbach, F. Moll, C. Fuchs, T. de Cola, R. Mata-Calvo "SPACE COMMUNICATIONS PROTOCOLS FOR FUTURE OPTICAL SATELLITE-DOWNLINKS”, 62nd International Astronautical Congress, Cape Town, South Africa, Oct. 2011

[5] D. Giggenbach, A. Shrestha, C. Fuchs, C. Schmidt, F. Moll, Florian, "SYSTEM ASPECTS OF OPTICAL LEO-TO-GROUND LINKS", International Conference on Space Optics (ICSO), 18-21 Okt. 2016, Biarritz, France

[6] L.C. Andrews, R.L. Phillips, "Laser Beam Propagation through Random Media, Second Edition", SPIE-Press, Bellingham, 2005

[7] F. Moll, "Experimental analysis of channel coherence time and fading behavior in the LEO-ground link," International Conference on Space Optical Systems and Applications. ICSOS 2014, 07.-09. May 2014, Kobe, Japan

[8] F. Moll, "Experimental characterization of intensity scintillation in the LEO downlink," 4th International Workshop on Optical Wireless Communications (IWOW), 2015.

[9] H. T. Yura, D. A. Kozlovski, "Low Earth orbit satellite-to-ground optical scintillation: comparison of experimental observations and theoretical predictions", Optics Letters Vol. 36 No. 13, July 2011

[10] F. Moll, A. Shrestha and C. Fuchs, "Ground stations for aeronautical and space laser communications at German Aerospace Center,” Proc. of SPIE 9647, 2015.

[11] F. Moll and M. Knapek, "Free-space laser communications for satellite downlinks: Measurements of the atmospheric channel,” International Astronautical Federation. Proceedings, 62nd International Astronautical Congress, Cape Town, South Africa, 3-7 Oct 2011 (2011), 2011.

[12] B. V. Oaida, M. J. Abrahamson, R. J. Witoff, J. N. B. Martinez, and D. A. Zayas, "OPALS: an optical communications technology demonstration from the International Space Station,” IEEE Aerospace Conference, 2013.

[13] A. Biswas, B. V. Oaida, K. S. Andrews, J. M. Kovalik, M. Abrahamsonand and M. W. Wright, "Optical payload for lasercomm science (OPALS) link validation during operations from the ISS,” 
Proc. of SPIE9354, Free-Space Laser Communication and Atmospheric Propagation XXVII, 2015.

[14] Y. Koyama, M. Toyoshima, Y. Takayama, H. Takenaka, K. Shiratama, I. Mase, and O. Kawamoto, "SOTA: Small Optical Transponder for micro-satellite”, Proceedings of ICSOS 2011, 2011.

[15] F. Moll, D. Kolev, M. Abrahamson, C. Schmidt, R. Mata Calvo and C. Fuchs, "LEO-ground scintillation measurements with the Optical Ground Station Oberpfaffenhofen and SOTA/OPALS space terminals”, Proc. of SPIE 9991, 2016.

[16] "Report on DLR-JAXA Joint Experiment - The Kirari Optical Downlink to Oberpfaffenhofen (KIODO)", published by Japan Aerospace Exploration Agency JAXA, ISSN 1349-1121, Tokyo, April 2007

[17] D. Giggenbach, "Optimierung der optischen Freiraumkommunikation durch die turbulente Atmosphäre - Focal Array Receiver”, Shaker Verlag 2005

[18] J.H. Churnside, ”Aperture averaging of optical scintillations in the turbulent atmosphere, Appl. Opt. Vol. 30, No. 15 / May 1991

[19] L.C. Andrews, “Aperture-averaging factor for optical scintillations of plane and spherical waves in the atmosphere”, J. Opt. Soc. Am. A, Vol. 9, No. 4 / April 1992

[20] L.C. Andrews, R.L. Phillips, and C.Young Hopen, “Aperture averaging of optical scintillations: power fluctuations and the temporal spectrum" Waves in Random and Complex Media 10, 53-70, 2000

[21] L.C. Andrews, R.L. Phillips, C. Young Hopen, "Scintillation model for a satellite communication link at large zenith angles" Optical Engineering 39 (12), 2000

[22] S.D. Lyke, D.G. Voelz, M.C. Roggemann, "Probability density of aperture-averaged irradiance fluctuations for long range free space optical communication links Appl. Opt., 2009, 48, 6511-6527

[23] F.S. Vetelino, C. Young, L.C. Andrews, J. Recolons, ”Aperture averaging effects on the probability density of irradiance fluctuations in moderate-to-strong turbulence”, Appl. Opt., 2007, 46, 2099-2108

[24] F.S. Vetelino, , S. Young, L.C. Andrews, "Fade statistics and aperture averaging for Gaussian beam waves in moderate-to-strong turbulence”, Appl. Opt., 2007, 46, 3780-3790

[25] N. Perlot, D. Fritzsche, “Aperture-Averaging - Theory and Measurements”, In: Proceedings of SPIE Vol. 5338, 2004

[26] D. Giggenbach, R. Mata-Calvo., "Sensitivity modeling of binary optical receivers,” Appl. Opt. 54, 8254-8259, 2015.

[27] D. Giggenbach, H. Henniger, "Fading-loss assessment in atmospheric free-space optical communication links with on-off keying”, Optical Engineering 47 (4), April 2008 